\title{
Spiral patterns beyond the optical radius: numerical simulations and synthetic HI observations
}

\author{
Sergey Khoperskov ${ }^{1,2}$ and Giuseppe Bertin ${ }^{3}$ \\ ${ }^{1}$ GEPI, Observatoire de Paris, CNRS, Université Paris Diderot, 5 place Jules Janssen, \\ 92190 Meudon, France \\ ${ }^{2}$ Institute of Astronomy, Russian Academy of Sciences, 48 Pyatnitskaya st., \\ 119017 Moscow, Russia email: sergey.khoperskov@obspm.fr \\ ${ }^{3}$ Dipartimento di Fisica, Università degli Studi di Milano, via Celoria 16, I-20133 Milano, Italy \\ email: giuseppe.bertin@unimi.it
}

\begin{abstract}
The outer parts of many galaxy disks exhibit extended spiral arms far beyond the optical radius. To understand the nature and the origin of such outer spiral structure, we investigate the propagation in the outer gaseous regions of large-scale spiral density waves excited in the bright optical disk. By means of 3D hydrodynamical simulations, we show that spiral density waves, penetrating in the gas through the outer Lindblad resonance, can indeed give rise to relatively regular patterns outside the bright optical stellar disk. The amplitude of spiral structure increases rapidly with radius. Beyond the optical radius, spirals become nonlinear and develop small-scale features related to shear-induced instabilities. We also construct the synthetic 21-cm data cubes extracted from simulated gaseous disks. Our synthetic HI observations point to the existence of specific kinematical features related to the presence of spiral pattern perturbations that should be found in deep HI observations.
\end{abstract}

Keywords. galaxies: kinematics and dynamics, galaxies: spiral, galaxies: structure

\section{Introduction}

Both extended ultraviolet disks and deep neutral hydrogen images frequently exhibit well-developed spiral arms far beyond the optical radius. For instance, GALEX images of NGC 1512 show long spirals extending out to $10-12$ optical scales (Koribalski \& Lopez-Sanchez 2009). A similar picture can be seen in deep 21-cm observations of NGC 4203 (Yıldız et al. 2015). Such extended structures are also detected in NGC 2915, NGC 5055, NGC 6744, NGC 6946, NGC 5236, NGC 7793 (Thilker et al. 2007, Boomsma et al. 2008, Bertin \& Amorisco 2010).

Grand-design spiral structure in galaxies is generally thought to be associated with density waves. In one scenario worked out in quantitative detail, it is argued that granddesign spiral structure results from the superposition of few, self-excited, long-lived global modes (Bertin 2014). Alternative scenarios are also reviewed by Bertin et al. (1989a), Bertin et al. (1989b), Bertin \& Lin (1996). In this paper we briefly summarize the results of our work on the origin and observational appearance of extended spiral arms on the galactic outskirts (Khoperskov \& Bertin 2015a,b); in this respect, we should mention that different mechanisms have also been proposed to explain the nature of the outer spiral structure, in particular tidal interactions (Bullock \& Johnston 2005) or interactions with a triaxial dark matter halo (Khoperskov et al. 2012, Butenko et al. 2015). 

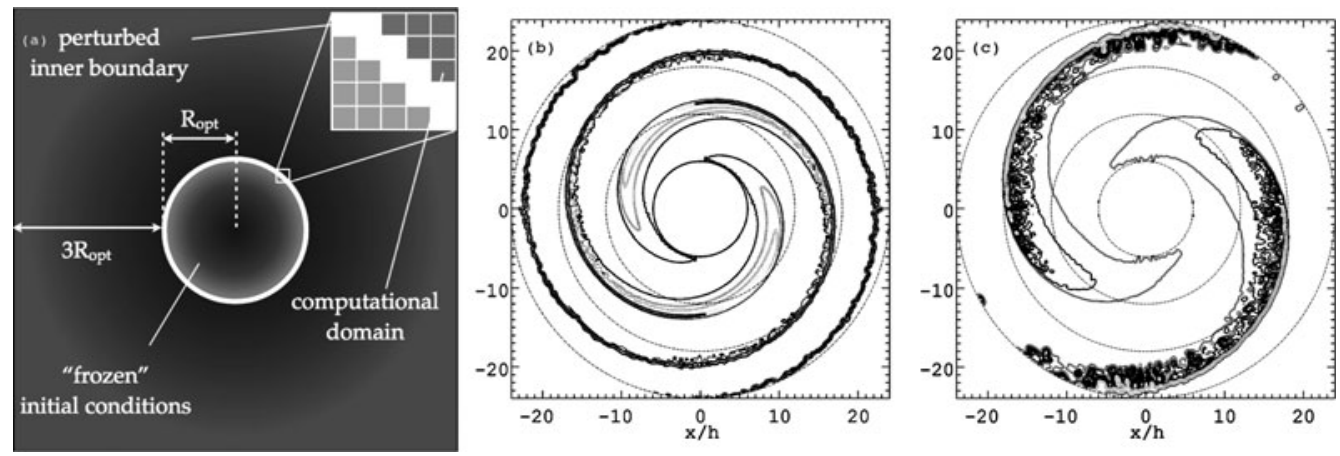

Figure 1. (a) The center circule indicates a fixed area at the outer boundary of which the initial conditions are imposed. The thin, light circule annulus, at the optical radius taken here to be $R_{o p t}=6 \mathrm{~h}$, is the location where the perturbation is imposed. The area outside the central circule is the actual computational domain. (b, c) The panels show the relative surface density perturbations for two models after several rotation periods. Contours vary from grey to black, which corresponds to values of the relative density perturbation in the range $0.01-1$.

\section{Numerical simulations and predictions on 21-cm observations}

By means of hydrodynamical simulations we have investigated the propagation in the outer gaseous disk of large-scale spiral waves excited in the bright optical disk. The general structure of the computational tools is described by Khoperskov et al. (2014). In the present paper we focus on the case in which the outer gaseous disk is dominated by a two-armed spiral pattern. Other examples with more complex morphologies can be found in a full-length journal article (Khoperskov \& Bertin 2015a). We consider density-wave perturbations of the hydrodynamical quantities at the inner boundary (cyan thin circular annulus in Fig. 1) with density proportional to $\cos (2 \theta-\omega t)$, where $t$ is the time and $\theta$ is the angular coordinate (see general scheme of the computational model in Fig. 1a).

The two-armed trailing spiral structure imposed at the inner boundary propagates outwards and its amplitude increases rapidly with radius. In our numerical simulations a quasi-stationary spiral structure is quickly established over the entire outer disk, well outside the bright optical disk (out to $6-20 \mathrm{~h}$, where $\mathrm{h}$ is the stellar disk scale length). For the simple case studied here, the gas surface density perturbation is shown in Fig. 1 b,c, where we illustrate two models with different initial gas velocity dispersion. More open spirals (right panel in Fig. 1) arise in disks with higher velocity dispersion. The amplitude of the perturbations increases with radius. At relatively small radii the structure remains in the low-amplitude regime and follows the linear theory developed by Bertin \& Amorisco (2010). At large radii the perturbations become nonlinear as marked by the presence of shocks and effects related to shear instabilities.

For a quantitative comparison with the observations we have computed the synthetic HI data cubes associated with our models (see Khoperskov \& Bertin 2015b). Simulated spectra show a clear signature of the kinematic perturbations induced by the presence of prominent spiral structure. We note a significant difference between the gas velocity dispersion in arm and inter-arm regions (see the corresponding line profiles in Fig. 2). Nonlinear spiral perturbations strongly affect the velocity field. The amplitude of the line-of-sight velocity perturbations increases with radius, marking the location of the spiral arms. Such systematic variations, if found in $21 \mathrm{~cm}$ observations, would give strong support to the physical scenario that we are considering (Bertin \& Amorisco 2010, Khoperskov \& Bertin 2015a,b). 

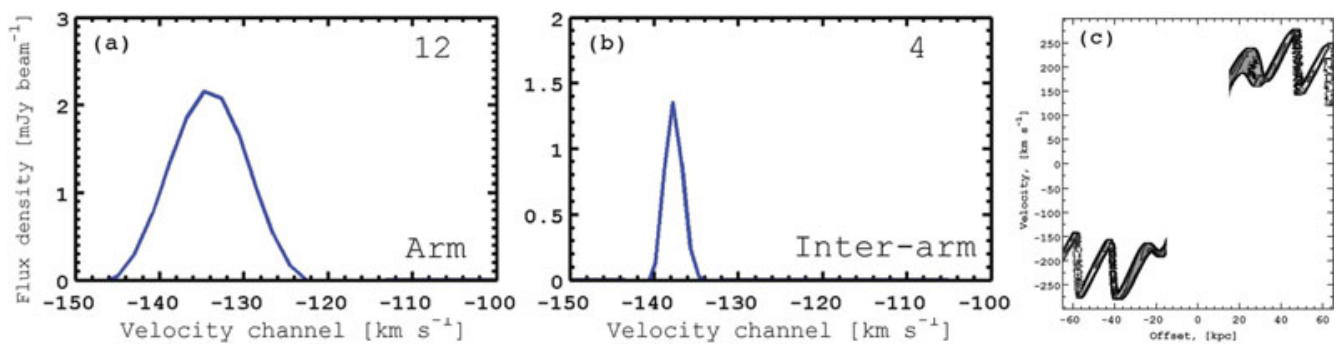

Figure 2. (a, b) Line profiles extracted from the HI data cube for a simulated gaseous disk. The estimated FWHM value is indicated in the top-right corner of each panel in units of $\mathrm{km} \mathrm{s}^{-1}$. (c) Position - velocity diagram along the major axis.

\section{Conclusions}

By means of hydrodynamical simulations we have shown that outside the bright optical stellar disk, regular spiral patterns in the gas can be explained as the natural counterpart of the dominant modes generated in the inner disk, which are expected to transfer angular momentum outwards by emitting short-trailing waves that can propagate through the outer Lindblad resonance. The amplitude of the perturbations increases with radius, forming prominent spiral patterns, as a sort of "galactic tsunami".

By constructing 21-cm synthetic observations, we have noted the establishment of significant spatial variations of the los-velocity dispersion around the disk: from 10$15 \mathrm{~km} \mathrm{~s}^{-1}$ in the arms down to $3-5 \mathrm{~km} \mathrm{~s}^{-1}$ in the inter-arm regions (see Fig. 2a,b). We have also found systematic deviations of the los-velocity with radius from $30 \mathrm{~km} \mathrm{~s}^{-1}$ up to $100 \mathrm{~km} \mathrm{~s}^{-1}$ with respect to the "normal" rotation curve characterizing the basic state (see Fig. 1c). Such features may be the objective of future observational projects related to the study of the outskirts of galaxy disks.

The numerical simulations have been performed at the RCC (MSU). This work was partially supported by the Italian MIUR, by the RF President grant (MK-4536.2015.2) and RFBR grants (15-02-06204, 15-52-12387).

\section{References}

Bertin, G., Lin, C. C., Lowe, S. A., \& Thurstans, R. P. 1989a, ApJ, 338, 78

Bertin, G., Lin, C. C., Lowe, S. A., \& Thurstans, R. P. 1989b, ApJ, 338, 104

Bertin, G. \& Lin, C. C. 1996 Spiral structure in galaxies: a density wave theory, The MIT Press, Cambridge, $M A$

Bertin, G. \& Amorisco, N. 2010, A\&A, 512, 17

Bertin, G. 2014, Dynamics of Galaxies, 2nd Ed. Cambridge University Press, New York

Boomsma, R., Oosterloo, T. A., Fraternali, F., van der Hulst, J. M., \& Sancisi, R. 2008, A\&্A, 490,555

Bullock, J. S. \& Johnston, K. V. 2005, ApJ, 635, 931

Butenko, M., Khoperskov, A., \& Khoperskov, S. 2015, Baltic Astronomy, 24, 119

Khoperskov, A. V., Eremin, M. A., Khoperskov, S. A., Butenko, M. A., \& Morozov, A. G. 2012, Astronomy Reports, 56, 16

Khoperskov, S. A., Vasiliev, E. O., Khoperskov, A. V., \& Lubimov, V. N. 2014, Journal of Physics Conference Series, 510 (1), 012011

Khoperskov, S. \& Bertin, G. 2015a, MNRAS, 451, 2889

Khoperskov, S. \& Bertin, G. 2015b, Journal of Plasma Physics, 81(6), 495810607

Koribalski, B. S. \& López-Sánchez, Á. R. 2015, MNRAS, 400, 1749

Thilker, D. A., et al., 2007, ApJS, 173, 538

Yıldız, M. K., Serra, P., Oosterloo, T. A., Peletier, R. F., Morganti, R., Duc, P.-A., Cuillandre, J.-C., \& Karabal, E. 2015, MNRAS, 451, 103 